\title{
Gestion Et Dispensation Des Medicaments Antiretroviraux A Brazzaville : Cas Du Centre De Traitement Ambulatoire (Cta) Et De La Pharmacie Du Centre Hospitalier Et Universitaire De Brazzaville (Chub)
}

\section{Mokoko Jean Bruno}

Faculté des Sciences et de la Santé, Université Marien Ngouabi, Pharmacie du Centre Hospitalier et Universitaire de Brazzaville, Congo

Ondélé Gandzaley

Pharmacie du Centre Hospitalier et Universitaire de Brazzaville, Congo

Abena Ange Antoine

Faculté des Sciences et de la Santé, Université Marien Ngouabi,

Laboratoire de Biochimie et de Pharmacologie, Congo

Diafouka MerlinNsondé Mahamboula

Centre de Traitement Ambulatoire de Brazzaville, Congo

Doi: 10.19044/esj.2018.v14n30p454 URL:http://dx.doi.org/10.19044/esj.2018.v14n30p454

\begin{abstract}
In Congo, the distribution of generic drugs including ARVs (Antiretrovirals) is done through dispensing sites. This paper focuses on describing the management, delivery, and supply of ARVs at two sites in Brazzaville, and thus contributes to improving the management of HIVpositive patients. This is a cross-sectional, observational, descriptive, and analytical study of 3,473 patients registered at the Hospital and University Hospital Pharmacy (CHUB) and Ambulatory Treatment Center (CTA) during the period from 1st January to December 31, 2010. The data were collected using medical records, prescriptions, inventory sheets, purchase orders, delivery notes of the Congolaise of Essential Medicines Generic (COMEG), and informal interview with the heads of dispensing units. Of the 3473 patients registered, there were 1412 men (40.65\%) and 2061 women (59.34\%). A sex ratio of 0.68 at the pharmacy of CHUB 13427 boxes of ARVs was ordered unlike the CTA where this number was 30810 boxes of ARV. The COMEG delivered to the CTA 28,667 boxes of ARVs (93.04\%) dispensing 18,994 boxes. Half of the ARVs dispensed were the triple combinations (56,48\%). At the CHUB pharmacy, COMEG delivered 15,371 boxes of ARVs, of which $28.16 \%$ is also a triple therapy. The analysis of the system revealed a poor
\end{abstract}


estimate of needs because the dispensing and management of these drugs does not conform to the active queue.

Keywords: Management, Dispensation, Antiretroviral drugs

\section{Résumé}

Au Congo, la distribution des médicaments génériques dont les ARV (Antirétroviraux), se fait par l'entremise des sites de dispensation. L'objectif du présent travail est de décrire la gestion, la dispensation et l'approvisionnement des ARV au sein de deux sites à Brazzaville et ainsi contribuer à l'amélioration de la prise en charge des PPVIH (Patients Porteurs du VIH). Il s'agit d'une étude transversale, observationnelle à visée descriptive et analytique, auprès de 3473 patients enregistrés à la Pharmacie du Centre Hospitalier et Universitaire (CHUB) et au Centre de Traitement Ambulatoire (CTA) au cours de la période allant du 1er janvier au 31 décembre 2010. Les données ont été recueillies à l'aide des : dossiers médicaux, ordonnances, fiches de stocks, bons de commande, bordereaux de livraison de la Congolaise des Médicaments Essentiels Générique (COMEG) et de l'entretien informel avec les responsables des unités de dispensation. Sur les 3473 patients enregistrés on comptait 1412 hommes $(40,65 \%)$ et 2061 femmes (59,34\%), soit un sex-ratio de 0,68. à la pharmacie du CHUB 13427 boîtes d'ARV ont été commandées contrairement au CTA où ce nombre était de 30810 boîtes d'ARV. La COMEG a livré au CTA 28667 boîtes d'ARV soient 93,04\% qui à dispenser 18994 boîtes, la moitié des ARV dispensés ont été les triples combinaisons soit 56,48\%. A la pharmacie du CHUB, la COMEG a livré 15371 boîtes d'ARV dont 28,16\% est aussi une trithérapie. L'analyse du système révèle une mauvaise estimation des besoins, car la dispensation et la gestion de ces médicaments n'est pas conforme à la file active.

Mots-clés : Gestion, Dispensation, Médicaments antirétroviraux

\section{Introduction}

A la fin de l'année 2008, l'Organisation Mondiale de la Santé (OMS) et I'ONUSIDA ont estimé que 33,4 millions de personnes vivaient avec le Virus de 1'Immunodéficience Humaine (VIH).En Afrique Subsaharienne, en 2005, 4700000 personnes infectées par le VIH avaient besoin d'un traitement antirétroviral (TARV) pour survivre. En près de dix ans, l'alliance des militants, des médecins et des chercheurs a rendu disponibles les ARV dans les pays en développement. En effet, on est passé de quelques centaines de malades traités en 2001 à plus de 4 millions à la fin de 2008 et certainement plus de 5 millions aujourd'hui (SIDACTION. Convention nationale, 2010). 
Ainsi, a-t-on observé un recul du nombre de décès grâce à un accès plus large aux antirétroviraux (ARV).Au Congo, en 2006, une centrale d'achat des médicaments génériques (COMEG) a été créée pour assurer la distribution des médicaments génériques, dont les ARV, à travers tout le pays par l'entremise des sites de dispensation et de prise en charge des personnes vivant avec le VIH (PVVIH) (Récépissé de déclaration d'association, 2005; Convention Gouvernement de la République du Congo-COMEG, 2006). Aussi, jusqu'en 2009, on estimait à environ 110000 les personnes vivant avec le VIH dans tout le pays dont 11525 adultes ont été mis sous traitement ARV (République du Congo, 2009), soit une couverture de $10 \%$ environ des besoins en ARV. L'objectif de ce travail était d'évaluer la gestion, la dispensation et l'approvisionnement des ARV au CTA et à la pharmacie du CHUB.

\section{Materiels et Méthodes}

Il s'est agi d'une étude observationnelle, transversale a visée descriptive et analytique, du système de gestion de stock et de dispensation des médicaments ARV au niveau de 2 sites de dispensation: le CTA et le CHUB, sur une période allant du $1^{\text {er }}$ janvier au 31 décembre 2010, soit 12 mois. La population cible était composée au niveau du CTA des patients enregistrés et s'y approvisionnant en médicaments ARV et au niveau de la pharmacie du CHUB de ceux qui venaient des services de gastro-entérologie (GEMI), maladies infectieuses (SMI), hématologie et pneumologie. Notre étude a inclus les patients âgés d'au moins 15 ans enregistrés dans les 2 sites de dispensation (CTA et pharmacie du CHUB) et y recevant le TARV. Il a été exclu de notre étude tous les patients âgés de moins 15 ans ne recevant pas ses ARV au CTA ou à la pharmacie du CHUB ainsi que les autres sites de dispensation privés, publics et confessionnels.

Les données ont été recueillies auprès de trois mille quatre cent soixante-treize (3473) patients enregistrés et suivis à l'aide des dossiers médicaux, des ordonnances, des fiches de stocks, des bons de commande, des bordereaux de livraison de la COMEG et de l'entretien informel avec les responsables des unités de dispensation des 2 sites.

Les variables étudiées ont été :

- Variables épidémiologiques : âge, sexe, centre de provenance.

- Variables liées aux types de VIH : VIH 1, VIH 2, VIH 1+2.

- Variables liées à la prise en charge thérapeutique.

- Système de dispensation.

- Dispensation des ARV.

- La gestion de stocks des médicaments ARV au niveau des 2 sites.

- La quantité des médicaments ARV commandée par les sites de dispensation à la COMEG durant notre période d'étude. 
- La quantité de médicaments ARV livrée par la COMEG durant notre période d'étude.

Les résultats sont présentés sous forme de commentaires et de tableaux. La saisie des données a été faite avec le logiciel Word et l'analyse des dites données avec le logiciel Excel.

\section{Résultats}

\section{II-1 : Données épidémiologiques}

\section{Age}

L'âge des patients était compris entre 30 et 44 ans avec des extrêmes de 15 et 65 ans (Tableau I).

Tableau I. Répartition des patients par tranche d'âge selon le site de dispensation

\begin{tabular}{|l|c|c|}
\hline \multicolumn{1}{|c|}{ Centre } & CTA & CHUB \\
\hline Tranche d'âge & & \\
\hline 15 à 29 ans & 147 & 164 \\
30 à 44 ans & 827 & 1187 \\
$>45$ ans & 736 & 412 \\
File active & $\mathbf{1 7 1 0}$ & $\mathbf{1 7 6 3}$ \\
\hline
\end{tabular}

\section{Sexe}

Sur les 3473 dossiers étudiés, on compte 1412 hommes, soit 40,65\% et 2061 femmes, soit 59,34\% pour un sex-ratio de 0,68 (Tableau II). Sur l'ensemble des patients 1710 proviennent du CTA, soit $49,23 \%$ et 1763 soit $50,76 \%$ proviennent de la pharmacie du CHUB (Tableau II).

Tableau II. Répartition des patients par sexe selon le site de dispensation

\begin{tabular}{|l|c|c|}
\hline \multicolumn{1}{|c|}{ Centre } & CTA & CHUB \\
\hline Sexe & & \\
\hline Masculin & 568 & 844 \\
Féminin & 1142 & 919 \\
File active & $\mathbf{1 7 1 0}$ & $\mathbf{1 7 6 3}$ \\
\hline
\end{tabular}

\section{II-2 Données liées au sérotype du VIH}

Tableau III. Répartition des patients par sérotype du VIH selon le centre de dispensation

\begin{tabular}{|l|c|c|}
\hline \multicolumn{1}{|c|}{ Centre } & CTA & CHUB \\
\hline Sérotype & & \\
\hline VIH 1 & 1700 & 1763 \\
VIH 2 & 0 & 0 \\
VIH $1+2$ & 10 & 0 \\
File active & $\mathbf{1 7 1 0}$ & $\mathbf{1 7 6 3}$ \\
\hline
\end{tabular}


III-Données liées à la prise en charge thérapeutique 1. Protocole d'ARV institué à l'initiation thérapeutique (Tableau IV)

Tableau IV. Répartition des patients par protocole d'ARV institué l'initiation du traitement.

\begin{tabular}{|l|c|c|}
\hline Centre & CTA & CHUB \\
\hline Protocole & & \\
\hline AZT+3TC+NVP & 985 & 1420 \\
AZT+3TC+EFV & 316 & 100 \\
D4T+3TC+EFV & 68 & 121 \\
D4T+3TC+NVP & 95 & 78 \\
DTF+FTC+EFV & 95 & 29 \\
ABC+3TC+EFV & 5 & 0 \\
DDI+3TC+EFV & 1 & 5 \\
Autres protocoles & 145 & 0 \\
Total & $\mathbf{1 7 1 0}$ & $\mathbf{1 7 6 3}$ \\
\hline
\end{tabular}

\section{Modifications de protocole d'ARV}

190 patients soit $5,47 \%$ ont changé de protocole thérapeutique :

$>$ Au CTA : 187 patients ont changé de protocole thérapeutique :

- 78 patients, soit $41,71 \%$, ont présenté des effets secondaires à la NVP ; celle-ci a été remplacée par l'EFV.

- 61 patients, soit 32,63\%, ont eu une intolérance à l'EFV, remplacée par la NVP.

- 48 patients, soit $25,63 \%$, ont eu une intolérance à la D4T qui a été remplacée par l'AZT.

$>$ A la pharmacie du CHUB : 3 patients ont eu une intolérance à $\mathrm{AZT}+3 \mathrm{TC}+\mathrm{EFV}$, cette association a été remplacée par FTC +TDF+EFV.

\section{Système de dispensation}

a) Types de dispensation utilisés en fonction des sites

La dispensation dans les 2 sites de prise en charge des PVVIH est nominative et individuelle.

\section{b) La présence d'un pharmacien}

Le CTA ne dispose pas de pharmacien dans le site de dispensation; par contre la pharmacie du CHUB à un pharmacien dispensateur.

c) Le Rythme d'approvisionnement des patients en ARV (Tableau V)

Tableau V. Rythme d'approvisionnement des patients en ARV au niveau des sites de dispensation

\begin{tabular}{|l|c|c|}
\hline Rythme d'approvisionnement & CTA & CHUB \\
\hline Tous les 6 mois & Non & Non \\
Chaque trimestre & Oui & Oui \\
Tous les mois & Non & Oui \\
Chaque semaine & Non & Non \\
\hline
\end{tabular}

A la pharmacie du CHUB, l'approvisionnement des patients a lieu en fonction de la durée du traitement prescrit par le médecin, et le nombre de 
traitements dispensés dépend de la quantité disponible en stock et au CTA. il correspond à trois (03) ou deux (02) mois s'il y a assez de stock et d'un (01) mois lorsque le stock est limité (Tableau V).

\section{d) Régularité des patients}

Dans les 2 sites il y a eu des patients réguliers, non réguliers et les perdus de vue (Tableau VI).

Tableau VI. Répartition des patients en fonction de la régularité d'approvisionnement dans

\begin{tabular}{|l|c|c|}
\multicolumn{3}{|c|}{ les sites de dispensation } \\
\hline Centre Régularité & CTA & CHUB \\
\hline Réguliers & 1460 & 1433 \\
Non réguliers & 111 & 0 \\
Perdue de vue & 139 & 330 \\
Dossiers étudiés & $\mathbf{1 7 1 0}$ & $\mathbf{1 7 6 3}$ \\
\hline
\end{tabular}

\section{Dispensation des ARV au niveau des 2 sites de priseen charge}

$\mathrm{Au}$ cours de notre étude, les dispensateurs n'ont pas rencontré des erreurs de prescriptions en tant que telles auprès des médecins prescripteurs.

Au niveau du CTA, lorsque le patient arrive à l'accueil, il présente un ticket qui précisera s'il s'agit d'un retrait d'ARV, d'une consultation clinique ou biologique ou d'un dépistage. Ensuite, on sort son dossier que l'on remet au médecin pour une consultation brève suivie d'une ordonnance dont une copie sera conservée par le médecin. Le dispensateur valide l'ordonnance et le nombre de boîtes nécessaires pour la durée du traitement qui varie d'un à trois mois.

A la pharmacie du CHUB, les consultations ont lieu dans différents services. Au moment de la dispensation, le malade présente son ordonnance ; la délivrance des boîtes de médicaments se fait après validation de celle-ci par le dispensateur et une copie lui est remise. Le dispensateur garde l'originale et il enregistre les références du malade (prescripteur, boîtes délivrées et date).

\section{Gestion des ARV aux niveaux des sites de dispensation}

$\mathrm{Au}$ CTA et à la pharmacie du CHUB, il n'y a pas eu de commande d'urgence. Dans chaque site, il y a un stock de sécurité de $20 \%$, toujours mis à jour par les responsables des centres de dispensation lors de la passation de commande. 
6. Approvisionnement en ARV aux niveaux des 2 sites de dispensation

A. Quantité d'ARV commandée par les 2 sites

a)Au niveau du CTA :

i. INTI (Inhibiteurs nucléosidiques de la transcriptase inverse) commandés au CTA (Tableau VII)

$>$ INTI forme isolée

Tableau VII. Récapitulatif de la quantité d'INTI forme isolée commandée par le CTA

\begin{tabular}{|l|c|c|c|c|c|}
\hline $\begin{array}{l}\text { Période } \\
\text { Nombre de boîtes }\end{array}$ & $\begin{array}{c}\mathbf{1}^{\text {er }} \\
\text { Trimestre }\end{array}$ & $\begin{array}{c}\mathbf{2}^{\text {ème }} \\
\text { Trimestre }\end{array}$ & $\begin{array}{c}\mathbf{3}^{\text {ème }} \\
\text { Trimestre }\end{array}$ & $\begin{array}{c}\mathbf{4}^{\text {ème }} \\
\text { Trimestre }\end{array}$ & Total \\
\hline ABC 300mg gel & 154 & 0 & 144 & 296 & 594 \\
AZT 300 mg cp & 140 & 0 & 0 & 0 & 140 \\
DDI 400mg cp & 200 & 0 & 0 & 50 & 250 \\
DDI 200 mg cp & 100 & 240 & 0 & 264 & 605 \\
3TC 150mg cp & 0 & 0 & 0 & 70 & 70 \\
D4T 30mg cp & 0 & 0 & 0 & 0 & 0 \\
TDF & 0 & 0 & 0 & 0 & 0 \\
Total & $\mathbf{5 9 4}$ & $\mathbf{2 4 0}$ & $\mathbf{1 4 4}$ & $\mathbf{6 8 0}$ & $\mathbf{1 6 5 8}$ \\
\hline
\end{tabular}

Au total mille six cent cinquante-huit (1658) boîtes d'INTI forme isolée ont été commandées. Par contre le TDF 200mg et le D4T 30mg n'ont pas été commandés par le CTA (Tableau VII).

INTIforme combinée (double combinaison)

Tableau VIII. Récapitulatif quantité INTI sous forme de double combinaison commandée

\begin{tabular}{|l|c|c|c|c|c|}
\hline Période & $\begin{array}{c}\mathbf{1}^{\text {er }} \\
\text { Nombre de boîtes }\end{array}$ & $\begin{array}{c}\mathbf{2}^{\text {ème }} \\
\text { Trimestre }\end{array}$ & $\begin{array}{c}\mathbf{3}^{\text {ème }} \\
\text { Trimestre }\end{array}$ & $\begin{array}{c}4^{\text {ème }} \\
\text { Trimestre }\end{array}$ & Total \\
\hline AZT +3TC (300+150) mg & 2360 & 2000 & 0 & 720 & 5080 \\
D4T+3TC (30+ 150) mg & 756 & 756 & 0 & 0 & 1512 \\
FTC+TDF (300+200) mg & 0 & 0 & 57 & 100 & 157 \\
Total & $\mathbf{3 1 1 6}$ & $\mathbf{2 7 5 6}$ & $\mathbf{5 7}$ & $\mathbf{8 2 0}$ & $\mathbf{6 7 4 9}$ \\
\hline
\end{tabular}

Six mille sept cent quarante-neuf (6749) boîtes d'INTI sous forme de double combinaison ont été commandées, avec 5080 boîtes de la double combinaison AZT + 3TC, forme la plus commandée parmi les INTI, dont 2360 boîtes au $1^{\mathrm{e}}$ Trimestre (Tableau VIII).

> INTI total : au total 8407 boîtes d'INTI ont été commandées durant la période d'étude (tableaux VII et VIII).

ii. INNTI (Inhibiteurs non nucléosidiques de la transcriptase inverse) commandés au CTA :

$>$ INNTI forme isolée 
Tableau IX. Récapitulatif de la quantité d'INNTI forme isolée commandée

\begin{tabular}{|l|c|c|c|c|c|}
\hline $\begin{array}{l}\text { Période } \\
\text { Nombre de boîtes }\end{array}$ & $\begin{array}{c}\mathbf{1}^{\text {er }} \\
\text { Trimestre }\end{array}$ & $\begin{array}{c}\mathbf{2}^{\text {ème }} \\
\text { Trimestre }\end{array}$ & $\begin{array}{c}\mathbf{3}^{\text {ème }} \\
\text { Trimestre }\end{array}$ & $\begin{array}{c}\mathbf{4}^{\text {ème }} \\
\text { Trimestre }\end{array}$ & Total \\
\hline NVP 200mg cp & 2510 & 1864 & 0 & 0 & 4374 \\
EFV 600mg cp & 765 & 1186 & 1500 & 0 & 341 \\
Total & $\mathbf{3 2 7 5}$ & $\mathbf{3 0 5 0}$ & $\mathbf{1 5 0 0}$ & 0 & $\mathbf{7 8 2 5}$ \\
\hline
\end{tabular}

Au total Sept mille huit cent vingt-cinq (7825) boîtes d'INNTI forme isolée ont été commandées, les commandes ont été nulles au $4^{\mathrm{e}}$ trimestre (Tableau IX).

INNTI triple combinaison

Tableau X. Récapitulatif de la quantité d'INNTI triple combinaison commandée

\begin{tabular}{|l|c|c|c|c|c|}
\hline $\begin{array}{l}\text { Période } \\
\text { Nombre de boîtes }\end{array}$ & $\begin{array}{c}\mathbf{1}^{\text {er }} \\
\text { Trimestre }\end{array}$ & $\begin{array}{c}\mathbf{2}^{\text {ème }} \\
\text { Trimestre }\end{array}$ & $\begin{array}{c}\mathbf{3}^{\text {ème }} \\
\text { Trimestre }\end{array}$ & $\begin{array}{c}\mathbf{4}^{\text {ème }} \\
\text { Trimestre }\end{array}$ & Total \\
\hline $\begin{array}{l}\text { FTC+TDF+EFV cp } \\
(200 / 300 / 600) \mathrm{mg}\end{array}$ & 40 & 55 & 387 & 346 & 828 \\
$\begin{array}{l}\text { AZT+3TC+NVP cp } \\
(300+150+200) \mathrm{mg}\end{array}$ & 410 & 3369 & 1577 & 6952 & 12308 \\
$\begin{array}{l}\mathrm{D} 4 \mathrm{TC}+3 \mathrm{TC}+\mathrm{NVP} \\
(30+150+200) \mathrm{mg}\end{array}$ & 0 & 0 & 0 & 0 & 0 \\
Total & $\mathbf{4 5 0}$ & $\mathbf{3 4 2 4}$ & $\mathbf{1 9 6 4}$ & $\mathbf{7 2 9 8}$ & $\mathbf{1 3 1 3 6}$ \\
\hline
\end{tabular}

Treize mille cent trente-six (13136) boîtes d'INNTI triple combinaison ont été commandées durant notre étude avec une prédominance de boite de la triple combinaison AZT + 3TC + NVP, notamment au 4e trimestre, soit 6952 boîtes commandées et un total de 12308 boîtes (Tableau X).

INNTI total : 20961 boîtes d'INNTI ont été commandées par le CTA durant notre période d'étude. Les triples combinaisons ont été les plus commandées (Tableaux X et XI).

iii. Les IP (Inhibiteurs de Protéase) commandés

Tableau XI. Récapitulatif de la quantité d'IP commandée

\begin{tabular}{|l|c|c|c|c|c|}
\hline $\begin{array}{l}\text { Période } \\
\text { Nombre de boîtes }\end{array}$ & $\begin{array}{c}\mathbf{1}^{\text {er }} \\
\text { Trimestre }\end{array}$ & $\begin{array}{c}\mathbf{2}^{\text {ème }} \\
\text { Trimestre }\end{array}$ & $\begin{array}{c}\mathbf{3}^{\text {ème }} \\
\text { Trimestre }\end{array}$ & $\begin{array}{c}\mathbf{4}^{\text {ème }} \\
\text { Trimestre }\end{array}$ & Total \\
\hline IDV 400mg ep & 0 & 0 & 0 & 0 & 0 \\
RTV100 mg cp & 100 & 0 & 0 & 0 & 100 \\
LPV/r cp (200+50) mg & 432 & 0 & 370 & 540 & 1342 \\
Total & $\mathbf{5 3 2}$ & 0 & $\mathbf{3 7 0}$ & $\mathbf{5 4 0}$ & $\mathbf{1 4 4 2}$ \\
\hline
\end{tabular}

Mille quatre cent quarante-deux (1442) boîtes d'IP ont été commandées par le CTA durant notre période d'étude, avec une prédominance du LPV/R ou Kaletra* soit 1342 boîtes (Tableau XI). 
iv. Quantité totale d'ARV commandée au CTA

Au total 30810 boîtes d'ARV ont été commandées par le CTA du 1 janvier au 31 décembre 2010 (Tableaux VII-XI). Les INNTI ont été les ARV les plus commandés durant notre période d'étude, soit 20961 boîtes (forte prédominance des triples combinaisons). De janvier à décembre 2010 les quantités commandées varient chaque trimestre. Le $4^{\mathrm{e}}$ trimestre a été celui où les commandes ont été les plus importantes (Tableaux VII-XI).

b) Au niveau de la pharmacie du $\mathrm{CHU}$

\section{i. INTI commandés à la pharmacie du CHU}

$>$ INTI forme isolée

Tableau XII. Récapitulatif de la quantité d'INTI forme isolé commandée

\begin{tabular}{|l|c|c|c|c|c|}
\hline Période & $\begin{array}{c}\mathbf{1}^{\text {er }} \\
\text { Nombre de boîtes }\end{array}$ & $\begin{array}{c}\mathbf{2}^{\text {ème }} \\
\text { Trimestre }\end{array}$ & $\begin{array}{c}\mathbf{3}^{\text {ème }} \\
\text { Trimestre }\end{array}$ & $\begin{array}{c}4^{\text {ème }} \\
\text { Trimestre }^{\text {Trimestre }}\end{array}$ & Total \\
\hline ABC 300mg cp & 29 & 100 & 100 & 40 & 269 \\
AZT 300mg cp & 0 & 179 & 378 & 50 & 607 \\
DDI 400mg cp & 18 & 150 & 50 & 40 & 258 \\
DDI 200mg cp & 28 & 60 & 36 & 40 & 164 \\
3TC 150mg cp & 0 & 0 & 420 & 256 & 676 \\
D4T 30mg cp & 11 & 0 & 0 & 30 & 41 \\
TDF 200mg cp & 0 & 15 & 0 & 0 & 15 \\
Total & $\mathbf{8 6}$ & $\mathbf{5 0 4}$ & $\mathbf{9 8 4}$ & $\mathbf{4 5 6}$ & $\mathbf{2 0 3 0}$ \\
\hline
\end{tabular}

Deux mille trente (2030) boîtes d'INTI forme isolée adulte ont été commandées. Au cours de notre période d'étude la 3TC a été I'INTI la plus commandée avec au total 676 boîtes. Le 3e trimestre est le trimestre où tous les INTI forme isolée Adulte ont été les plus commandés, soit 984 boîtes en tout (Tableau XII).

\section{INTI double combinaison}

Tableau XIII. Récapitulatif de la quantité d'INTI double combinaison commandée

\begin{tabular}{|l|c|c|c|c|c|}
\hline $\begin{array}{l}\text { Période } \\
\text { Nombre de boîtes }\end{array}$ & $\begin{array}{c}\mathbf{1}^{\text {er }} \\
\text { Trimestre }\end{array}$ & $\begin{array}{c}\mathbf{2}^{\text {ème }} \\
\text { Trimestre }\end{array}$ & $\begin{array}{c}\mathbf{3}^{\text {ème }} \\
\text { Trimestre }\end{array}$ & $\begin{array}{c}\mathbf{4}^{\text {ème }} \\
\text { Trimestre }\end{array}$ & Total \\
\hline D4T +3TC cp (30+150) mg & 276 & 100 & 330 & 115 & 821 \\
AZT+3TCcp (300+150)mg & 493 & 800 & 1010 & 830 & 3133 \\
FTC+TDE cp (300+200) mg & 4 & 0 & 150 & 50 & 204 \\
Total & $\mathbf{7 7 3}$ & $\mathbf{9 0 0}$ & $\mathbf{1 4 9 0}$ & $\mathbf{9 9 5}$ & $\mathbf{4 1 5 8}$ \\
\hline
\end{tabular}

Quatre mille cent cinquante-huit (4158) boîtes d'INTI double combinaison ont été commandées. Il y a eu de forte commande d'INTI au $3^{\mathrm{e}}$ trimestre, soit 1490 boîtes au total (Tableau XIII). 
INTI total : au total 6188 boîtes d'INTI ont été commandées par la pharmacie du CHU durant notre période d'étude.

\section{ii. INNTI commandée}

\section{INNTI forme isolée}

Tableau XIV. Récapitulatif de la quantité d'INNTI forme isolée commandée

\begin{tabular}{|l|c|c|c|c|c|}
\hline Période & $\begin{array}{c}\mathbf{1}^{\text {er }} \\
\text { Nombre de boîtes }\end{array}$ & $\begin{array}{c}\mathbf{2}^{\text {ème }} \\
\text { Trimestre }\end{array}$ & $\begin{array}{c}\mathbf{3}^{\text {ème }} \\
\text { Trimestre }\end{array}$ & $\begin{array}{c}\mathbf{4}^{\text {ème }} \\
\text { Trimestre }\end{array}$ & Total \\
\hline NVP cp 200mg & 516 & 455 & 560 & 235 & 1766 \\
EFV cp 600mg & 0 & 0 & 0 & 0 & 0 \\
EFV cp 200mg & 0 & 0 & 0 & 0 & 0 \\
Total & $\mathbf{5 1 6}$ & $\mathbf{4 5 5}$ & $\mathbf{5 6 0}$ & $\mathbf{2 3 5}$ & $\mathbf{1 7 6 6}$ \\
\hline
\end{tabular}

Mille sept cent soixante-six (1766) boîtes d'INNTI forme adulte isolée ont été commandées, par contre EFV $600 \mathrm{mg}$ et $200 \mathrm{mg}$ n'ont pas été commandés. La NVP cp $200 \mathrm{mg}$ a été la plus commandée, 560 boîtes au 3 trimestre pour un total de 1766 (Tableau XIV).

\section{INNTI triple combinaison}

Tableau XV: Récapitulatif de la quantité d'INNTI triple combinaison commandées

\begin{tabular}{|c|c|c|c|c|c|}
\hline $\begin{array}{l}\text { Période } \\
\text { Nombre de boîtes }\end{array}$ & $\begin{array}{c}1^{\mathrm{er}} \\
\text { Trimestre }\end{array}$ & $\begin{array}{c}2^{\text {ème }} \\
\text { Trimestre }\end{array}$ & $\begin{array}{c}3^{\text {ème }} \\
\text { Trimestre }\end{array}$ & $\begin{array}{c}4^{\text {ème }} \\
\text { Trimestre }\end{array}$ & Total \\
\hline $\begin{array}{l}\text { FTC+TDF-t-EFV } \\
(200) 300 / 600) \mathrm{mg} \mathrm{cp}\end{array}$ & 14 & 20 & 110 & 130 & 274 \\
\hline $\begin{array}{l}\mathrm{D} 4 \mathrm{~T}+3 \mathrm{TC}+\mathrm{NVP} \\
(30 / 150 / 200) \mathrm{mg} \mathrm{cp}\end{array}$ & 793 & 0 & 280 & 180 & 1253 \\
\hline $\begin{array}{l}\text { AZT }+3 T C+N V P \\
(300 / 150 / 200) \mathrm{mg} \mathrm{cp}\end{array}$ & 630 & 560 & 709 & 1300 & 3199 \\
\hline Total & 1437 & 580 & 1099 & 1610 & 4726 \\
\hline
\end{tabular}

Quatre mille sept cent vingt-six (4726) boîtes d'INNTI triple combinaison ont été commandées. La triple combinaison AZT+3TC+NVP (3199 boîtes) a été la plus commandée durant notre période d'étude. Ainsi, un total de 1610 boîtes de toutes les triples combinaisons ont été commandées au $4^{\text {ème }}$ trimestre (Tableaux XV).

INNTI total : 6492 boîtes d'INNTI ont été commandées par la pharmacie du CHUB. Notons que durant notre période d'étude, les triples combinaisons ont été les plus commandées avec au total 4726 boîtes (Tableaux XIV et XV).

\section{iii. Les IP commandés}


Tableau XVI. Récapitulatif de la quantité d'IP commandés

\begin{tabular}{|l|c|c|c|c|c|}
\hline Période & $\begin{array}{c}\mathbf{1}^{\text {er }} \\
\text { Nombre de boîtes }\end{array}$ & $\begin{array}{c}\mathbf{2}^{\text {ème }} \\
\text { Trimestrestre }\end{array}$ & $\begin{array}{c}\mathbf{3}^{\text {ème }} \\
\text { Trimestre }\end{array}$ & $\begin{array}{c}\mathbf{4}^{\text {ème }} \\
\text { Trimestre }\end{array}$ & Total \\
\hline IDV 400mg cp & 18 & 4 & 15 & 37 & 37 \\
RTV100mgcp & 0 & 0 & 0 & 30 & 30 \\
LPv/r 200mg cp & 290 & 0 & 240 & 150 & 680 \\
Total & $\mathbf{3 0 8}$ & $\mathbf{4}$ & $\mathbf{2 5 5}$ & $\mathbf{1 8 0}$ & $\mathbf{7 4 7}$ \\
\hline
\end{tabular}

Sept cent quarante-sept (747) boîtes d'IP ont été commandées. Le $\mathrm{LPV} / \mathrm{r}$ (Aluvia) a été le plus commandé, par contre le SQV, qui est également un IP forme adulte présent à la COMEG, n'a pas été commandé par la pharmacie du CHUB durant notre période d'étude (Tableau XVI).

iv. Quantité totale d'ARV commandée par la pharmacie du CHUB

Au total, 13427 boîtes d'ARV ont été commandées par la pharmacie du CHU. Les INTI ont été les ARV les plus commandés avec 6188 boîtes durant notre période d'étude. Notons que ces quantités d'ARV commandées varient chaque trimestre. Les commandes les plus importantes ont eu lieu au $3^{\text {ème }}$ trimestre. Le $4^{\text {ème }}$ trimestre est celui où peu de commandes ont été passées hormis les triples combinaisons d'INNTI qui ont été beaucoup commandées (Tableaux XII-XVI).

\section{B. Quantités d'ARV livrées par la COMEG}

a. Quantités d'ARV livrées par la COMEG au CTA durant notre période d'étude

i. INTI livrés

$>$ INTI forme isolée

Tableau XVII. Récapitulatif de la quantité d'INTI livrés forme isolée

\begin{tabular}{|l|c|c|c|c|c|}
\hline $\begin{array}{l}\text { Période } \\
\text { Nombre de boite }\end{array}$ & $\begin{array}{c}\mathbf{1}^{\text {er }} \\
\text { Trimestre }\end{array}$ & $\begin{array}{c}\mathbf{2}^{\text {ème }} \\
\text { Trimestre }\end{array}$ & $\begin{array}{c}\mathbf{3}^{\text {ème }} \\
\text { Trimestre }\end{array}$ & $\begin{array}{c}4^{\text {ème }} \\
\text { Trimestre }\end{array}$ & Total \\
\hline ABC cp 300mg & 154 & 0 & 144 & 296 & 594 \\
DDI gel 400mg & 200 & 0 & 0 & 0 & 200 \\
DDI 200 mg cp & 100 & 240 & 0 & 267 & 607 \\
3TC 150 mg cp & 0 & 0 & 0 & 70 & 70 \\
AZT cp 300mg & 140 & 0 & 0 & 0 & 140 \\
D4T cp 30mg & 0 & 0 & 0 & 0 & 0 \\
TDF cp200mg & 0 & 0 & 0 & 0 & 0 \\
Total & $\mathbf{5 9 4}$ & $\mathbf{2 4 0}$ & $\mathbf{1 4 4}$ & $\mathbf{6 3 3}$ & $\mathbf{1 6 1 1}$ \\
\hline
\end{tabular}

Au total 1611 boîtes d'INTI forme isolée adulte ont été livrées, et seulement 144 boîtes d'INTI ont été livrées durant tout le 3ème trimestre, ces 144 boîtes représentant les boîtes d'ABC cp 300 mg (Tableau XVII). 
INTI double combinaison

Tableau XVIII. Récapitulatif de la quantité d'INTI double combinaison livrée

\begin{tabular}{|l|c|c|c|c|c|}
\hline Période & $\begin{array}{c}\mathbf{1}^{\text {er }} \\
\text { Molécules }\end{array}$ & $\begin{array}{c}\mathbf{2}^{\text {ème }} \\
\text { Trimestre }\end{array}$ & $\begin{array}{c}\mathbf{3}^{\text {ème }} \\
\text { Trimestre }\end{array}$ & $\begin{array}{c}\mathbf{4}^{\text {ème }} \\
\text { Trimestre }\end{array}$ & Total \\
\hline FTC+TDF cp (200+300) mg & 0 & 0 & 57 & 100 & 157 \\
D4T+3TC cp (30+150) mg & 756 & 756 & 0 & 0 & 1512 \\
AZT+3TC cp(300+150) mg & 2360 & 2000 & 0 & 3116 & 5080 \\
Total & $\mathbf{3 1 1 6}$ & $\mathbf{2 7 5 6}$ & $\mathbf{5 7}$ & $\mathbf{8 2 0}$ & $\mathbf{6 7 4 9}$ \\
\hline
\end{tabular}

Six mille sept cent quarante-neuf (6749) boîtes d'INTI double combinaison ont été livrées par la COMEG au CTA. La double combinaison AZT +3 TC cp a été le plus livrée (5080 boîtes), par contre D4T+3TC n'a pas été livrée pendant les deux derniers trimestres (Tableau XVIII).

Total INTI : 8360boîtes d'INTI ont été livrées par la COMEG durant notre période d'étude. Les doubles combinaisons ont été les plus livrées.

ii. INNTI livrés

INNTI forme isolée

Tableau XIX. Récapitulatif de la quantité d'INNTI livrée

\begin{tabular}{|l|c|c|c|c|c|}
\hline Période & $\begin{array}{c}\mathbf{1}^{\text {er }} \\
\text { Nombre de boite }\end{array}$ & $\begin{array}{c}\mathbf{2}^{\text {ème }} \\
\text { Trimestre }\end{array}$ & $\begin{array}{c}\mathbf{3}^{\text {ème }} \\
\text { Trimestre }\end{array}$ & $\begin{array}{c}4^{\text {ème }} \\
\text { Trimestre }\end{array}$ & Total \\
\hline NVP cp 300mg & 2510 & 1864 & 0 & 0 & 4374 \\
EFVcp 600mg & 0 & 1156 & 1500 & 0 & 2656 \\
EFVcp200mg & 0 & 0 & 30 & 0 & 30 \\
Total & $\mathbf{2 5 1 0}$ & $\mathbf{3 0 2 0}$ & $\mathbf{1 5 3 0}$ & 0 & $\mathbf{7 0 6 0}$ \\
\hline
\end{tabular}

Sept mille soixante (7060) boîtes d'INNTI ont été livrées. Aucune livraison d'INNTI n'a été effectuée au 4ème trimestre (Tableau XIX).

\section{INNTI triple combinaison}

Tableau XX. Récapitulatif de la quantité d'INNTI triple combinaison

\begin{tabular}{|l|c|c|c|c|c|}
\hline $\begin{array}{l}\text { Période } \\
\text { Nombre de boite }\end{array}$ & $\begin{array}{c}\mathbf{1}^{\text {er }} \\
\text { Trimestre }\end{array}$ & $\begin{array}{c}\mathbf{2}^{\text {ème }} \\
\text { Trimestre }\end{array}$ & $\begin{array}{c}\mathbf{3}^{\text {ème }} \\
\text { Trimestre }\end{array}$ & $\begin{array}{c}4^{\text {ème }} \\
\text { Trimestre }\end{array}$ & Total \\
\hline $\begin{array}{l}\text { FTC+TDF+EF cp } \\
(200+300+600) \mathrm{mg}\end{array}$ & 40 & 55 & 287 & 0 & 382 \\
$\begin{array}{l}\text { D4T+3TC+NVP cp } \\
(30+150+200) \mathrm{mg}\end{array}$ & 0 & 1090 & 0 & 0 & 1090 \\
AZT+3TC+NVP & 223 & 3384 & 50 & 6952 & 10609 \\
Cp (300+150+200) mg & $\mathbf{2 6 3}$ & $\mathbf{4 5 2 9}$ & $\mathbf{3 3 7}$ & $\mathbf{6 9 5 2}$ & $\mathbf{1 2 0 8 1}$ \\
Total & & & & & \\
\hline
\end{tabular}

Douze mille quatre-vingt et une (12081) boîtes d'INNTI combinaison ont été livrées par la COMEG. Les triples combinaisons FTC+TDF+EFV et $\mathrm{D} 4 \mathrm{~T}+3 \mathrm{TC}+\mathrm{NVP}$ n'ont pas été livrées au $4^{\text {ème }}$ trimestre (Tableau XX). 
Total INNTI : 19441 boîtes d'INNTI ont été livrées avec un grand nombre d'INNTI triple combinaison livrée, soit 12081 boîtes (Tableaux XIX et XX).

\section{iii. Les IP livrés}

Tableau XXI. Récapitulatif de la quantité d'IP livrés

\begin{tabular}{|l|c|c|c|c|c|}
\hline $\begin{array}{l}\text { Période } \\
\text { Nombre de boîtes }\end{array}$ & $\begin{array}{c}\mathbf{1}^{\text {er }} \\
\text { Trimestre }\end{array}$ & $\begin{array}{c}\mathbf{2}^{\text {ème }} \\
\text { Trimestre }\end{array}$ & $\begin{array}{c}\mathbf{3}^{\text {ème }} \\
\text { Trimestre }\end{array}$ & $\begin{array}{c}\mathbf{4}^{\text {ème }} \\
\text { Trimestre }\end{array}$ & Total \\
\hline LPV/r cp(200+50 mg) & 432 & 0 & 94 & 540 & 1066 \\
RTV cp 100mg & 100 & 0 & 0 & 0 & 100 \\
Total & $\mathbf{5 3 2}$ & 0 & $\mathbf{9 4}$ & $\mathbf{5 4 0}$ & $\mathbf{1 1 6 6}$ \\
\hline
\end{tabular}

Mille cent soixante-six (1166) boîtes d'IP forme adulte ont été livrées. Le RTV cp n' a été livré qu'au $1^{\text {er }}$ trimestre, soit 100 boîtes (Tableau XXI).

iv. Quantité totale ARV livrés par COMEG au CTA : 28667 boîtes livrées

b. Quantité d'ARV livrés par la COMEG à la pharmacie du CHUB

i. INTI livrés

$>$ INTI forme isolée

Tableau XXII. Récapitulatif de la quantité d'INTI forme isolée livrée

\begin{tabular}{|l|c|c|c|c|c|}
\hline $\begin{array}{l}\text { Période } \\
\text { Nombre de boîtes }\end{array}$ & $\begin{array}{c}\mathbf{1}^{\text {er }} \\
\text { Trimestre }\end{array}$ & $\begin{array}{c}\mathbf{2}^{\text {ème }} \\
\text { Trimestre }\end{array}$ & $\begin{array}{c}\mathbf{3}^{\text {ème }} \\
\text { Trimestre }\end{array}$ & $\begin{array}{c}\mathbf{4}^{\text {ème }} \\
\text { Trimestre }\end{array}$ & Total \\
\hline ABC300mgcp & 29 & 221 & 0 & 40 & 290 \\
AZT300mgcp & 0 & 179 & 378 & 50 & 607 \\
DDJ400mg gel & 18 & 150 & 50 & 40 & 258 \\
DDIcp200mg & 0 & 60 & 36 & 40 & 136 \\
DDI 100 mg cp & 28 & 0 & 0 & 0 & 28 \\
3TC300mg cp & 0 & 0 & 420 & 256 & 676 \\
D4T30 mg cp & 11 & 0 & 0 & 30 & 41 \\
TDF200mgcp & 0 & 15 & 0 & 0 & 15 \\
Total & $\mathbf{8 6}$ & $\mathbf{6 2 5}$ & $\mathbf{8 8 4}$ & $\mathbf{4 5 6}$ & $\mathbf{2 0 5 1}$ \\
\hline
\end{tabular}

Deux mille cinquante et une (2051) boîtes d'INTI ont été livrées. La $3 T C \mathrm{cp} 300 \mathrm{mg}$ a été la molécule la plus livrée, soit 676 boîtes, mais elle n'a pas été livrée au cours des deux premiers trimestres. La DDI $100 \mathrm{mg} \mathrm{cp}$ n'a pas été livrée durant les 3 derniers trimestres et la D4T $30 \mathrm{mg} \mathrm{cp}$ au $2^{\text {ème }}$ et $3^{\text {ème }}$ trimestre (Tableau XXII).

\section{INTI double combinaison}

Tableau XXIII. Récapitulatif de la quantité d'INTI double liaison livrée

\begin{tabular}{|l|c|c|c|c|c|}
\hline Période & $\begin{array}{c}\mathbf{1}^{\text {er }} \\
\text { Nombre de boîtes }\end{array}$ & $\begin{array}{c}\mathbf{2}^{\text {ème }} \\
\text { Trimestre }\end{array}$ & $\begin{array}{c}\mathbf{3}^{\text {ème }} \\
\text { Trimestre }\end{array}$ & $\begin{array}{c}\mathbf{4}^{\text {ème }} \\
\text { Trimestre }\end{array}$ & Total \\
\hline D4T-1-3TC cp (30+150) mg & 276 & 100 & 330 & 115 & 821 \\
AZT+3TC cp (300+15) mg & 493 & 800 & 960 & 905 & 3158 \\
FTC+TDF cp (200+300) mg & 4 & 0 & 150 & 110 & 264 \\
Total & $\mathbf{7 7 3}$ & $\mathbf{9 0 0}$ & $\mathbf{1 4 4 0}$ & $\mathbf{1 1 3 0}$ & $\mathbf{4 2 4 3}$ \\
\hline
\end{tabular}


Quatre mille deux cent quarante-trois (4243) boîtes d'INTI double combinaison ont été livrées par la COMEG. Cependant, la double combinaison AZT+3TC (Duovir) a été la plus livrée, soit 3158 boîtes dont 960 au $3^{\text {ème }}$ trimestre (Tableau XXIII).

ii. INNTI livré

INNTI forme isolée

Tableau XXIV. Récapitulatif de la quantité d'INNTI forme isolée livrée

\begin{tabular}{|l|c|c|c|c|c|}
\hline $\begin{array}{l}\text { Période } \\
\text { Nombre de boîtes }\end{array}$ & $\begin{array}{c}\mathbf{1}^{\text {er }} \\
\text { Trimestre }\end{array}$ & $\begin{array}{c}\mathbf{2}^{\text {ème }} \\
\text { Trimestre }\end{array}$ & $\begin{array}{c}\mathbf{3}^{\text {ème }} \\
\text { Trimestre }\end{array}$ & $\begin{array}{c}\mathbf{4}^{\text {ème }} \\
\text { Trimestre }\end{array}$ & Total \\
\hline NVP cp 200 mg & 516 & 555 & 840 & 435 & 2346 \\
EFVcp600mg & 0 & 294 & 0 & 286 & 580 \\
Total & $\mathbf{5 1 6}$ & $\mathbf{8 4 9}$ & $\mathbf{8 4 0}$ & $\mathbf{7 2 1}$ & $\mathbf{2 9 2 6}$ \\
\hline
\end{tabular}

Deux mille neuf cent vingt-six (2926) boîtes d'INNTT forme isolée adulte ont été livrées. La NVP cp $200 \mathrm{mg}$ a été la plus livrée et l'EFV cp 600rng n'a pas été livré aux $1^{\text {er }}$ et $3^{\text {ème }}$ trimestres (Tableau XXIV).

\section{$>$ INNTI triple combinaison}

Tableau XXV. Récapitulatif de la quantité d'INNTI triple combinaison livrée

\begin{tabular}{|l|c|c|c|c|c|}
\hline Période & $\begin{array}{c}\mathbf{1}^{\text {er }} \\
\text { Trimestre }\end{array}$ & $\begin{array}{c}\mathbf{2}^{\text {ème }} \\
\text { Trimestre }\end{array}$ & $\begin{array}{c}\mathbf{3}^{\text {ème }} \\
\text { Trimestre }\end{array}$ & $\begin{array}{c}\mathbf{4}^{\text {ème }} \\
\text { Trimestre }\end{array}$ & Total \\
\hline FTC+TDF+EFV cp(200+300+600)mg & 72 & 20 & 110 & 130 & 332 \\
D41+3IU+FVl cp (30+150+200) mg & 793 & 0 & 280 & 180 & 1253 \\
AZT+3TC+NVPcp(300+150+200) mg & 630 & 560 & 709 & 1900 & 3799 \\
Total & $\mathbf{1 4 9 5}$ & $\mathbf{5 8 0}$ & $\mathbf{1 0 9 9}$ & $\mathbf{2 2 1 0}$ & $\mathbf{5 3 8 4}$ \\
\hline
\end{tabular}

Cinq mille trois cent quatre-vingt-quatre (5384) boîtes d'INNTI triple combinaison ont été livrées. L'AZT-3TC+NVP cp a été la plus livrée, 3799 boîtes, dont 1900 au $4^{\text {ème }}$ trimestre (Tableau XXV).

\section{iii. Les IP livrés}

Tableau XXVI. Récapitulatif de la quantité d'IP livrée par la COMEG

\begin{tabular}{|l|c|c|c|c|c|}
\hline $\begin{array}{l}\text { Période } \\
\text { Nombre de boîtes }\end{array}$ & $\begin{array}{c}\mathbf{1}^{\text {er }} \\
\text { Trimestre }\end{array}$ & $\begin{array}{c}\mathbf{2}^{\text {ème }} \\
\text { Trimestre }\end{array}$ & $\begin{array}{c}\mathbf{3}^{\text {ème }} \\
\text { Trimestre }\end{array}$ & $\begin{array}{c}4^{\text {ème }} \\
\text { Trimestre }\end{array}$ & Total \\
\hline IDV cp 400mg & 22 & 0 & 15 & 30 & 67 \\
RTV cp 100 mg & 0 & 0 & 0 & 30 & 30 \\
LPV/r (200+50) mg & 290 & 0 & 240 & 140 & 670 \\
Total & $\mathbf{3 1 2}$ & 0 & $\mathbf{2 5 5}$ & $\mathbf{2 0 0}$ & $\mathbf{7 6 7}$ \\
\hline
\end{tabular}

Sept cent soixante-sept (767) boîtes d'IP ont été livrées, dont 670 boîtes LPV/r. Aucune livraison n'a été faite au 2e trimestre et le RTV cp 100 mg n'a pas été livré au cours des 3 premiers trimestres (Tableau XXVI). 
Quantités d'ARV livrées par la COMEG: 15371 boîtes d'ARV qui ont été livrées par la COMEG au niveau de la pharmacie du CHU (Tableau XXII-XXVI).

iv. Quantités d'ARV dispensées au niveau des 2 sites de dispensation a. Quantités d'ARV dispensées au CTA durant notre période d'étude

i. INTI dispensés

INTI forme isolée

Tableau XXVII. Récapitulatif de la quantité d'INTI forme isolée dispensée

\begin{tabular}{|l|c|c|c|c|c|}
\hline $\begin{array}{l}\text { Période } \\
\text { Nombre de boîtes }\end{array}$ & $\begin{array}{c}\mathbf{1}^{\text {er }} \\
\text { Trimestre }\end{array}$ & $\begin{array}{c}\mathbf{2}^{\text {ème }} \\
\text { Trimestre }\end{array}$ & $\begin{array}{c}\mathbf{3}^{\text {ème }} \\
\text { Trimestre }\end{array}$ & $\begin{array}{c}4^{\text {ème }} \\
\text { Trimestre }\end{array}$ & Total \\
\hline ABC 300mg cp & 55 & 187 & 81 & 271 & 591 \\
DDI 400 mg gel & 12 & 142 & 46 & 0 & 200 \\
DDI 200mg cp & 0 & 0 & 0 & 51 & 51 \\
3TC 150 mg cp & 18 & 7 & 12 & 33 & 70 \\
D4T 30 mg cp & 8 & 1 & 0 & 0 & 9 \\
AZT 300 mg cp & 10 & 0 & 1 & 72 & 83 \\
Total & $\mathbf{1 0 3}$ & $\mathbf{3 3 7}$ & $\mathbf{1 4 0}$ & $\mathbf{4 2 7}$ & $\mathbf{1 0 0 7}$ \\
\hline
\end{tabular}

Mille sept (1007) boîtes d'INTI forme isolée dispensées. L'ABC cp $300 \mathrm{mg}$ a été le plus dispensé avec une totalité de 594 boîtes, dont 271 au $4{ }^{\text {ème }}$ trimestre. La DDI 200mg cp n'a pas été dispensée durant les trois premiers trimestres (Tableau XXVII).

\section{ii. INTI forme double combinaisons dispensée}

Tableau XXVIII. Récapitulatif de la quantité d'INTI forme double combinaisons dispensée

\begin{tabular}{|l|c|c|c|c|c|}
\hline $\begin{array}{l}\text { Période } \\
\text { Nombre de boîtes }\end{array}$ & $\begin{array}{c}\mathbf{1}^{\text {er }} \\
\text { Trimestre }\end{array}$ & $\begin{array}{c}\mathbf{2}^{\text {ème }} \\
\text { Trimestre }\end{array}$ & $\begin{array}{c}\mathbf{3}^{\text {ème }} \\
\text { Trimestre }\end{array}$ & $\begin{array}{c}4^{\text {ème }} \\
\text { Trimestre }\end{array}$ & Total \\
\hline $\begin{array}{l}\text { FTC+TDF cp } \\
(200+300) \text { mg }\end{array}$ & 1 & 26 & 22 & 108 & 157 \\
$\begin{array}{l}\text { D4T+3TC cp } \\
(30+150) \mathrm{mg}\end{array}$ & 116 & 147 & 151 & 270 & 684 \\
AZT + 3TC cp & & & & & \\
$(300+150) \mathrm{mg}$ & 824 & 678 & 849 & 387 & 2738 \\
Total & $\mathbf{9 4 1}$ & $\mathbf{8 5 1}$ & $\mathbf{1 0 2 2}$ & $\mathbf{7 6 5}$ & $\mathbf{3 5 7 9}$ \\
\hline
\end{tabular}

Trois mille cinq cent soixante-dix neuf (3579) boîtes d'INTI double combinaison ont été dispensées. La double combinaison AZT+3TC cp a été la plus dispensée avec 2738 boîtes au total, soit 849 boîtes au $3^{\text {e }}$ trimestre (Tableau XXVIII). 
INTI Total : 4589 boîtes d'INTI ont été dispensées au CTA durant notre période d'étude, avec une prédominance des INTI double combinaison représentée par 3579 boîtes (Tableaux XXVII et XXVIII).

\section{iii. INNTI dispensée}

\section{INNTI forme isolée dispensée}

Tableau XXIX. Récapitulatif de la quantité d'INNTI forme isolée dispensée

\begin{tabular}{|l|c|c|c|c|c|}
\hline Période & $\begin{array}{c}\mathbf{1}^{\text {er }} \\
\text { Nombre de boîtes }\end{array}$ & $\begin{array}{c}\mathbf{2}^{\text {ème }} \\
\text { Trimestre }\end{array}$ & $\begin{array}{c}\mathbf{3}^{\text {'̀me }} \\
\text { Trimestre }\end{array}$ & $\begin{array}{c}4^{\text {ème }} \\
\text { Trimestre }\end{array}$ & Total \\
\hline NVP cp 300mg & 8 & 0 & 0 & 46 & 54 \\
EFV cp 600mg & 522 & 563 & 759 & 824 & 2670 \\
Total & $\mathbf{5 3 0}$ & $\mathbf{5 6 3}$ & $\mathbf{7 5 9}$ & 872 & $\mathbf{2 7 2 4}$ \\
\hline
\end{tabular}

Deux mille sept cent vingt-quatre (2724) boîtes d'INNTI forme isolée ont été dispensées. La NVP cp $300 \mathrm{mg}$ n'a pas été dispensée aux $2^{\text {ème }}$ et $3^{\text {ème }}$ trimestres (Tableau XXIX).

\section{INNTI forme triple dispensée}

Tableau XXX. Récapitulatif de la quantité d'INNTI forme triple dispensée

\begin{tabular}{|l|c|c|c|c|c|}
\hline $\begin{array}{l}\text { Période } \\
\text { Nombre de boîtes }\end{array}$ & $\begin{array}{c}\mathbf{1}^{\text {er }} \\
\text { Trimestre }\end{array}$ & $\begin{array}{c}\mathbf{2}^{\text {ème }} \\
\text { Trimestre }\end{array}$ & $\begin{array}{c}\mathbf{3}^{\text {ème }} \\
\text { Trimestre }\end{array}$ & $\begin{array}{c}4^{\text {ème }} \\
\text { Trimestre }\end{array}$ & Total \\
\hline $\begin{array}{l}\text { FTC+TDF+EFV cp } \\
(200+300+600) \mathrm{mg}\end{array}$ & 26 & 38 & 94 & 224 & 382 \\
$\begin{array}{l}\text { D4T+3TC+NVP cp } \\
(30+150+200) \mathrm{mg}\end{array}$ & 479 & 216 & 203 & 130 & 1028 \\
AZT+3TC+NVP cp & & & & & \\
$(300+150+200) \mathrm{mg}$ & 2671 & 2492 & 2768 & 1388 & 9319 \\
Total & $\mathbf{3 1 7 6}$ & $\mathbf{2 7 4 6}$ & $\mathbf{3 0 6 5}$ & $\mathbf{1 7 4 2}$ & $\mathbf{1 0 7 2 9}$ \\
\hline
\end{tabular}

Dix mille sept cent vingt-neuf (10729) boîtes d'INNTI triple combinaison ont été dispensées La triple combinaison AZT+3TC+NVP a été la plus dispensée durant notre période d'étude avec un total de 9319 boîtes, soit 2768 boîtes au $3^{\text {ème }}$ trimestre (Tableau XXX).

INNTI total: Au total13453 boîtes d'INNTI ont été dispensées par le CTA durant la période d'étude.

iv. Les IP dispensées

Tableau XXXI. Récapitulatif de la quantité des IP dispensées

\begin{tabular}{|l|c|c|c|c|c|}
\hline $\begin{array}{l}\text { Période } \\
\text { Nombre de boîtes }\end{array}$ & $\begin{array}{c}\mathbf{1}^{\text {er }} \\
\text { Trimestre }\end{array}$ & $\begin{array}{c}\mathbf{2}^{\text {ème }} \\
\text { Trimestre }\end{array}$ & $\begin{array}{c}\mathbf{3}^{\text {ème }} \\
\text { Trimestre }\end{array}$ & $\begin{array}{c}\mathbf{4}^{\text {ème }} \\
\text { Trimestre }\end{array}$ & Total \\
\hline LPV/r cp(200+50) mg & 143 & 255 & 289 & 206 & 893 \\
RTV cp 100mg & 0 & 4 & 2 & 0 & 6 \\
IDV cp 400mg & 15 & 15 & 7 & 19 & 56 \\
Total & $\mathbf{1 5 8}$ & $\mathbf{2 7 4}$ & $\mathbf{2 9 8}$ & $\mathbf{2 2 5}$ & $\mathbf{9 5 5}$ \\
\hline
\end{tabular}


Neuf cent cinquante-cinq (955) boîtes d'IP forme adulte ont été dispensées. Le LPV/r cp a été le plus dispensé au CTA, 893 boîtes au total pour 289 boîtes au $3^{\text {ème }}$ trimestre (Tableau XXXI).

v. Total d'ARV dispensé par le CTA: 18994 boîtes d'ARV ont été dispensées par le CTA durant notre période d'étude au CTA, les triples combinaisons, soit 56,48\%, représentent la moitié des ARV dispensés ; par contre les IP ont été dispensés en très faible quantité $(5,02 \%)$ au cours de l'année 2010 (Tableaux XXVII-XXXI).

b. Quantités d'ARV dispensées par la pharmacie du CHU

i. INTI dispensés

INTI forme isolée

Tableau XXXII. Récapitulatif de la quantité d'INTI forme isolée dispensée

\begin{tabular}{|l|c|c|c|c|c|}
\hline $\begin{array}{l}\text { Période } \\
\text { Nombre de boîte }\end{array}$ & $\begin{array}{c}\mathbf{1}^{\text {er }} \\
\text { Trimestre }\end{array}$ & $\begin{array}{c}\mathbf{2}^{\text {ème }} \\
\text { Trimestre }\end{array}$ & $\begin{array}{c}\mathbf{3}^{\text {ème }} \\
\text { Trimestre }\end{array}$ & $\begin{array}{c}4^{\text {ème }} \\
\text { Trimestre }\end{array}$ & Total \\
\hline ABC300mgcp & 0 & 0 & 37 & 8 & 45 \\
DDI400mg gel & 13 & 15 & 0 & 13 & 41 \\
DDI200mgcp & 1 & 30 & 0 & 1 & 32 \\
3TCcp150mg & 43 & 0 & 0 & 30 & 73 \\
AZT300mgep & 10 & 45 & 10 & 1 & 66 \\
D4T cp30mg & 30 & 10 & 0 & 1 & 41 \\
Total & $\mathbf{9 7}$ & $\mathbf{1 0 0}$ & $\mathbf{4 7}$ & $\mathbf{5 4}$ & $\mathbf{2 9 8}$ \\
\hline
\end{tabular}

Deux cent quarante vingt dix-huit (298) boîtes ont été dispensées avec un grand nombre de 3TC, soient 73 boîtes, avec 43 boîtes au $1^{\mathrm{e}}$ trimestre et 30 boîtes au $4^{\mathrm{e}}$ trimestre (Tableau XXXII).

\section{INTI forme combinée (double combinaison)}

Tableau XXXIII. Récapitulatif de la quantité d'INTI double combinaison dispensée

\begin{tabular}{|l|c|c|c|c|c|}
\hline Période & $\begin{array}{c}\mathbf{1}^{\text {er }} \\
\text { Nombre de boîte }\end{array}$ & $\begin{array}{c}\mathbf{2}^{\text {ème }} \\
\text { Trimestre }\end{array}$ & $\begin{array}{c}\mathbf{3}^{\text {(̀me }} \\
\text { Trimestre }\end{array}$ & $\begin{array}{c}4^{\text {ème }} \\
\text { Trimestre }\end{array}$ & Total \\
\hline FTC+TDF cp (200+300) mg & 6 & 15 & 0 & 68 & 89 \\
D4T+3TC cp (30+I50) mg & 315 & 90 & 0 & 83 & 488 \\
AZT+3TC cp (300+150)mg & 150 & 246 & 0 & 315 & 711 \\
Total & $\mathbf{4 7 1}$ & $\mathbf{3 5 1}$ & 0 & $\mathbf{4 6 6}$ & $\mathbf{1 2 8 8}$ \\
\hline
\end{tabular}

1288 boîtes d'INTI double combinaison ont été dispensées. Il n'y a pas eu de dispensation de toutes les doubles combinaisons au $3^{\text {ème }}$ trimestre (Tableau XXXIII).

Total INTI : 1586 boîtes d'INTT ont été dispensées à la pharmacie du CHU. Les

INTI double combinaison ont été les plus dispensés (Tableaux XXXIIXXXIII). 


\section{ii. Les INNTI dispensées}

INNTI forme isolée

Tableau XXXIV. Récapitulatif de la quantité d'INNTI forme isolée dispensée.

\begin{tabular}{|l|c|c|c|c|c|}
\hline Période & $\begin{array}{c}\mathbf{1}^{\text {er }} \\
\text { Nombre de boîtes }\end{array}$ & $\begin{array}{c}\mathbf{2}^{\text {ème }} \\
\text { Trimestre }\end{array}$ & $\begin{array}{c}\mathbf{3}^{\text {ème }} \\
\text { Trimestre }\end{array}$ & $\begin{array}{c}\mathbf{4}^{\text {ème }} \\
\text { Trimestre }\end{array}$ & Total \\
\hline VPcp200nig & 117 & 82 & 0 & 103 & 302 \\
EFV cp600 mg & 0 & 0 & 0 & 243 & 243 \\
EFVcp200mg & 60 & 0 & 0 & 10 & 70 \\
Total & $\mathbf{1 7 7}$ & $\mathbf{8 2}$ & 0 & $\mathbf{3 5 6}$ & $\mathbf{6 1 5}$ \\
\hline
\end{tabular}

Six cent quinze (615) boîtes d'INNTI forme isolée ont été dispensées. Les INNTI n'ont pas été dispensés durant tout le $3^{\mathrm{e}}$ trimestre. En plus, l'EFV cp $600 \mathrm{mg}$ n'a pas été dispensé au cours des 3 trimestres ; l'EFV cp $200 \mathrm{mg}$ aux $2^{\text {ème }}$ et $3^{\text {ème }}$ trimestres (Tableau XXXIV).

\section{INNTI triple combinaison}

Tableau XXXV. Récapitulatif de la quantité d'INNTI triple combinaison dispensée

\begin{tabular}{|l|c|c|c|c|c|}
\hline Période & $\begin{array}{c}\mathbf{1}^{\text {er }} \\
\text { Nombre de boîtes }\end{array}$ & $\begin{array}{c}\mathbf{2}^{\text {ème }} \\
\text { Trimestre }\end{array}$ & $\begin{array}{c}\mathbf{3}^{\text {ème }} \\
\text { Trimestre }\end{array}$ & $\begin{array}{c}4^{\text {ème }} \\
\text { Trimestre }\end{array}$ & Total \\
\hline FTC+TDF+EFVcp (200+300+600)mg & 11 & 27 & 0 & 48 & 86 \\
D4TC+3TC+NVP cp (30+150+200)mg & 180 & 165 & 0 & 316 & 661 \\
AZT+3TC+NVP cp (300+150+200)mg & 0 & 329 & 0 & 1045 & 1374 \\
Total & $\mathbf{1 9}$ & $\mathbf{5 2 1}$ & 0 & $\mathbf{1 4 0 9}$ & $\mathbf{2 1 2 1}$ \\
\hline
\end{tabular}

Deux mille cent vingt et une (2121) boîtes d'INNTI triple dispensées. Aucune triple combinaison n'a été dispensée combinaison ont été au $3^{\mathrm{e}}$ trimestre. La AZT+3TC+NVP a été beaucoup dispensée, 1374 boîtes au total, avec 1045 boîtes au 4emeTrimestre (Tableau XXXV).

\section{iii. Total INNTI}

Au total2736 boîtes d'INNTI ont été dispensées par la pharmacie du CHU.

iv. Les IP dispensés

Tableau XXXVI. Récapitulatif de la quantité d'IP dispensée

\begin{tabular}{|l|c|c|c|c|c|}
\hline Période & $\begin{array}{c}\mathbf{1}^{\text {er }} \\
\text { Nombre de boîtes }\end{array}$ & $\begin{array}{c}\mathbf{2}^{\text {ème }} \\
\text { Trimestre }\end{array}$ & $\begin{array}{c}\mathbf{3}^{\text {ème }} \\
\text { Trimestre }\end{array}$ & $\begin{array}{c}4^{\text {ème }} \\
\text { Trimestre }\end{array}$ & Total \\
\hline LPV/r cp (200+50) mg & 58 & 110 & 290 & 72 & 530 \\
IDVcp 400 mg & 16 & 0 & 2 & 1 & 19 \\
RTVcp 100 mg & 0 & 7 & 0 & 0 & 7 \\
Total & $\mathbf{7 4}$ & $\mathbf{1 1 7}$ & $\mathbf{2 9 2}$ & $\mathbf{7 3}$ & $\mathbf{5 5 6}$ \\
\hline
\end{tabular}

556 boîtes d'IP ont été dispensées, avec une prédominance du LPV/r cp représentée par 530 boîtes, soit 290 au 3e trimestre. Les boîtes d'IDV et de RTV cp n'ont presque pas été dispensées (Tableau XXXVI). 


\section{v. Quantité totale d'ARV dispensés à la pharmacie du CHUB}

4878 boîtes d'ARV ont été dispensées par la pharmacie du CHUB durant notre étude, et le tiers des ARV dispensés sont les triples combinaisons soient 28,88\% (Tableaux XXXII-XXXVI).

\section{Discussion}

Le choix de cette étude se justifie par l'ampleur de la pandémie du VIH SIDA, laquelle constitue un véritable problème de santé publique. L'OMS estime que près de deux tiers (63\%) de l'ensemble des personnes vivant avec le VIH se trouvent en Afrique subsaharienne, soit 24,7 millions (21,8 millions-27,7 millions) (Agence Universitaire De La Francophonie, 2009) et le nombre de décès dans notre région s'élève à 2,1 millions ( 1,8 million- 2,4 millions), ce qui représente $72 \%$ du total mondial des décès dus au SIDA (Deslaux, 2003). A cela, s'ajoute dans les pays en voie de développement la difficulté d'accès des malades aux ARV. Dans notre échantillon de 3473 dossiers étudiés, 57,99 \% des patients sont compris dans la tranche d'âge de « 30 à 44 ans ». A Bamako, dans une étude de Niangaly la tranche d'âge comprise entre 30-39 ans était représentée à 37,1\% en 2006 (Niangaly, 2007). Ces résultats montrent que l'infection à VIH touche plus les adultes en activité de reproduction comme l'avaient retrouvé Yhya Dieye en Mauritanie (Yayha, 2009), Haidara au Mali (Haidara Ramatoulaye, 2006) et d'autres résultats similaires qui rapportent une prédominance des jeunes adultes (Garraitv Moliwa, 2001; Ischrive \& Balleereau, 1995).

Nos dossiers étudiés comptaient 1412 hommes soit 40,65\% et 2061 femmes soit 59,34\%, pour un sex-ratio de 0,68 en faveur des femmes. Au Mali, les travaux de thèse de Niangaly retrouvent $54,7 \%$ de femmes contre $45,3 \%$ d'hommes avec un sexe ratio de 1,20 en faveur des femmes (Niangaly, 2007). Cette prédominance féminine du taux des personnes sous traitement antirétroviral est conforme avec les tendances nationales et internationales de la prévalence d'infection à VIH. Au Sénégal, les travaux de Ndiaye le confirment avec un sex-ratio de 0,8 (Ndiaye, 2004) et au Bénin, les travaux de Aplogan ainsi que ceux de Dormont. Un groupe d'experts à Paris montrent également une féminisation du nombre des personnes sous traitement antirétroviral avec un sex ratio de 0,66 (Yeman Colombe Aplogan, 2008) et 1,25 (Dormont, 1998). D'autres auteurs ont retrouvé une domination féminine avec des sex ratio de 1,03 et 2,19 (Cholie, Kakou \& Zephra, 1999; Elchdl Wua \& Chaissonr Moorer, 1998). Avant la création de la centrale, l'approvisionnement des ARV étaient l'apanage de plusieurs entités (Programme Nationale de Lutte Contre le VIH Sida, Direction des Études et de la Planification au Ministère de la Santé). Or, selon l'ACAME, la multiplicité des acteurs dans la chaîne de distribution est à l'origine des difficultés au niveau de la détermination des besoins, ainsi qu'une dilution des 
responsabilités de chaque entité (CNLS, 2005). En 2003 et 2004 le Burkina Faso ainsi que le Cameroun avaient inclus les ARV dans le circuit d'approvisionnement des médicaments essentiels (Karim, 2008). Au Congo, la dispensation des ARV est décentralisée, mais ils ont intégré ce circuit en 2006 avec la création de la COMEG. Les différents centres de dispensation sont catégorisés en trois : les centres privés, les centres publics et les centres confessionnels.

Dans les 2 sites de prise en charge des PPVVIH étudiés la dispensation était nominative et individuelle, ce qui est conforme aux normes nationales (OMS/République du Congo/Ministère de la Santé et des Affaires Sociales, 2008). De plus elle est journalière. Le CTA, centre privé qui ne dispose pas de pharmacien dans le site de dispensation à l'opposé de la pharmacie du CHUB qui a un pharmacien. Selon le code de la déontologie pharmaceutique (REMED, 2011), la présence d'un pharmacien est indispensable dans une officine privée, comme dans les pharmacies des centres de santé et hôpitaux publics. Les fiches de gestion de stocks étaient présentes dans les deux sites. Or, la présence d'une fiche de stock est indispensable pour que les conditions minimales de gestion des stocks soient atteintes (République du Sénégal/ Ministère de la Santé e de la Prévention/DPL/OMS, 2009).

Les patients viennent s'approvisionner à la pharmacie du CHUB en fonction de la durée du traitement prescrit par le médecin et le nombre de traitements dispensés est fonction de la quantité de stock disponible au niveau de la pharmacie. Au CTA 1'approvisionnement est trimestriel. Les travaux de Aplogan montrent qu'au CNHU Hubert Koutoucou Maga de Cotonou la dispensation est mensuelle (Yeman Colombe Aplogan, 2008). La régularité des patients est de 85,38 \% au CTA, de 81,28\% à la pharmacie du CHUB ce qui est comparable aux travaux de Y.C. APLOGAN au Béninavec83,21\% et de Dieye en Mauritanie 84\% (Yayha, 2009; Yeman Colombe Aplogan, 2008). Les perdus de vue représentent au CTA un 9,28\% et à la pharmacie du CHUB 19,45\%, alors qu'au Bénin, en Mauritanie et au Mali ils sont respectivement de 7,83\%; 17,1\% et de 16\% (Yayha, 2009; Yeman Colombe Aplogan, 2008). Cela peut s'expliquer par des décès non déclarés, le recours à la pharmacopée traditionnelle, les changements de centres, les voyages.

Au Congo, la plupart des classes thérapeutiques des médicaments ARV sont disponibles en dehors de celle des inhibiteurs de fusion et d'intégrasse.

Sur les 2 sites enquêtés lors de notre étude, il y a eu une commande d'urgence au CTA lorsqu'il y a une erreur de livraison (livraison au CTA d'un dosage autre que celui qui est demandé). Par ailleurs, le site peut commettre une erreur sur l'estimation des besoins pour couvrir un trimestre. Dans chacun des 2 sites, il y a un stock de sécurité de $20 \%$, toujours mis de côté par les responsables des sites de dispensation lors de la passation de commande. Ce 
stock permet au site de continuer à fonctionner entre le moment de la passation de commande et la livraison (République du Congo, 2000).

Au total, 8407 boîtes d'INTI ont été commandées par le CTA, dont 6749 boîtes représentent les doubles combinaisons d'INTI avec une prédominance de l'association AZT+3TC (5080 boîtes). Sur ces boîes INT commandées, 8360 ont été livrés et $54,54 \%$ soient 4586 ont été dispensées. Concernant les 13136 boîtes d'INNTI commandées, l'AZT+3TC+NVP est la trithérapie représentée à 93,09\%, soient 12308 boites. A la pharmacie du CHUB, 6188 boites d'INTI ont été commandées durant notre période d'étude et sur les 4158 boites de double combinaison, 3133 boites commandées étaient l'association AZT+3TC. Aussi, sur 6492 boîtes INNT, 4726 sont des boîtes de triple combinaison d'INNTI et de même qu'au CTA, la trithérapie la plus représentée est AZT+3TC+NVP (3199 boites soit 72,79\%). Ces résultats au niveau des 2 sites qui montrent la prédominance de AZT+3TC+NVP sont proches de ceux de Konaté en 2005 au Mali avec 79\% mais différents de ceux de Niangaly en 2007 et de Sanogo en 2007 qui avaient eu respectivement 59\% et $84,35 \%$ de patients sous $3 \mathrm{TC}+\mathrm{d} 4 \mathrm{~T}+\mathrm{NVP}$. Au niveau des 2 sites, les IP ont été les moins commandées par rapport aux autres classes INTI et INNTI, car au Congo ils sont utilisés en cas de VIH2 en association avec 2INTI+IP (Republique du Congo, 2010).

La COMEG a livré au CTA 28667 boîtes d'ARV pour une commande de 30810, soit un indice de satisfaction globale (ISG) de 93,04\%. Cependant, seules 18994 ont été dispensées (INTI 4586 boîtes d'ARV dispensées contre 8360 boîtes livrées par la COMEG, 13453 boîtes d'INNTI dispensées contre 19141 livrés). A la pharmacie du CHUB 15371 boîtes d'ARV ont été livrées pour une commande de 13427 avec un ISG de 114,47\% (INTI 1588 boîtes d'ARV dispensés contre 6294 boites livrées par la COMEG, 2736 boîtes d'INNTI dispensées contre 8310). Parmi les conséquences de cette dispensation insuffisante figurent les péremptions ; à la pharmacie du CHUB, 1257 boites d'ARV étaient périmées soit 8,17\%. Au contraire, au CTA les ARV dont les dates de péremptions approchaient étaient envoyés vers d'autres sites afin de les aider à écouler les molécules.

\section{Conclusion}

$\mathrm{Au}$ terme de cette étude transversale à visée analytique et descriptive, 3473 patients ont été inclus avec une prédominance féminine. Il en ressort que sur les 30810 boîtes que le CTA avait commandé seuls 18994 boites avaient été dispensées avec une prédominance des trithérapies. De la même manière, la pharmacie du CHUB alors qu'elle avait commandé 13427 boîtes ARV, la COMEG a livré 15371(un surplus de 1944 boîtes non justifiés) ; la forme la plus dispensée était également la trithérapie. 
L'analyse du système de dispensation permet de conclure que les médicaments ARV sont délivrés selon les règles pharmaceutiques nationales et internationales. Cependant, la gestion au niveau des sites ne répond pas aux exigences pharmaceutiques car au CTA il n'y a pas de pharmacien dispensateur. A la pharmacie du CHUB il y a un écart entre la file active et la quantité livrée, et les quantités dispensées n'atteignent pas également la moitié de celles livrées par la COMEG pour la pharmacie du CHUB et pour le CTA un peu plus de la moitié des ARV livrés.

Ce travail prouve que certaines dispositions doivent être prises en ce qui concerne la dispensation et la gestion des ARV, à savoir la sensibilisation des gestionnaires à l'importance de la bonne tenue de fiches de stock, une bonne estimation des besoins pour éviter le sur stockage et les péremptions des ARV et le recrutement d'un pharmacien si possible pour le CTA.

\section{References:}

1. Agence Universitaire De La Francophonie (2009). Dictionnaire universel Hachette Edicef 5è édition.

2. Cholie, S.P., Kakou, A.R., \& Zephra (1999). Association infect à VIH tuberculose : cause de décentralisation du cahier Santé; 1-6.

3. Convention Gouvernement de la République du Congo-COMEG (2006).

4. Deslaux, A. (2003). ARV en Afrique : de la culture dans une économie mondialisée. Anthropologie et Société 2003 ; vol 27 ; n॰2 : P4.

5. Dormont, J. (1998). Groupe d'experts. Nouveaux antirétroviraux et hydroxyurée in stratégie d'utilisations d'ARV dans l'infection par le VIH. Rapport1998, Ministre de l'emploi et de la solidarité. Paris : Flammarion; 37-41.

6. CNLS (2005). «Rapport de situation sur la riposte nationale à l'épidémie de VIH/SIDA. Senegal».

7. Elchdl Wua \& Chaissonr Moorer (1998). Adherence to antiretroviral and pneumocystis prophylaxis in disease. JAIDS; 1117-25.

8. Garraitv Moliwa, J.M. (2001). Nouvelles stratégies de traitement antirétroviral chez les personnes infectées par le VIH. Pathol. Biol.; 67-71.

9. Haidara (Ramatoulaye) (2006). Etude de l'observance aux antirétroviraux dans le service des maladies infectieuses à l'HNPG à propos de 270 cas. Bamako : université de Bamako, FMPOS, 200690f-Annexe. Thèse : Médecine.

10. Ischrive, S. \& Balleereau, F. (1995). Les médicaments du Sida. Paris : Marketing SA: 384p.

11. Karim, D. (2008). Intégration des ARV dans le circuit des médicaments essentiels : un modèle pour améliorer l'accès aux soins 
CAS DU SENEGAL. Mémoire Master 2 Santé Publique. Université Victor Segalen Bordeaux 2 / Institut de Santé Publique et de Développement.

12. Ndiaye (2004). Effets secondaires de la thérapie antirétrovirale de la prise en charge des personnes vivants avec le VIH/SIDA : Expérience sénégalaise. Thèse de médecine, Dakar, $\mathrm{N}^{\circ} 41$

13. Niangaly (2007). Evaluation de l'observance des anti retro viraux chez les patients suivis à l'hôpital Somine Dolo de Mopoti. Thèse de pharm., Bamako.

14. OMS/République du Congo/Ministère de la Santé et des Affaires Sociales (2008). Table ronde sur la problématique des médicaments au Congo. Juin, p 22.

15. Ousmane Sanogo (2007). Dispensation des Antirétroviraux à l'hôpital régional Nianankoro Fomba de Ségou. Thèse : pharmacie, FMPOS, Bamako; $\mathrm{N}^{\circ} 38$

16. Récépissé de déclaration d'association (2005). Ministère de la décentralisation et de l'administration du territoire/Direction générale de l'administration du territoire/ Direction des études et de la réglementation/ Service des affaires générales, 30/09/2005.

17. REMED (2011). Bien dispenser les médicaments, www.remed.org.Site consulté le 12 Mai.

18. République du Congo (2000). Ministère de la santé, des affaires sociales et de la famille/DGS Manuel de gestion des médicaments dans les hôpitaux de références, $\mathrm{P}$ 13-14.

19. Republique du Congo (2010). MSP/DGS/PNLS, Guide thérapeutique de la prise en charge du VIH/SIDA. Révisé octobre, chapitre 4 : les coinfections. P 30.

20. République du Sénégal/ Ministère de la Santé e de la Prévention/DPL/OMS (2009). Cartographie et évaluation approfondies des systèmes d'approvisionnement et de distribution des médicaments essentiels et autres produits de santé au Sénégal, P37.

21. République du Congo (2009). MSP/PNLS. Guide thérapeutique de prise en charge du VIH/sida au Congo, P 3.

22. SIDACTION. Convention nationale (2010). Collection les essentiels de Sidaction: monde au milieu du gué. www.sidaction.org site consulté le 23 décembre 2010.

23. Yayha, D. (2009). Gestion et dispensation des médicaments antirétroviraux en Mauritanie : cas du centre de traitement ambulatoire du CHN de Nouakchott. Thèse de pharm., Dakar, №79.

24. Yeman Colombe Aplogan (2008). Dispensation des antirétroviraux au Bénin : Cas du CNHU Hubert Koutoucou Maga de Cotonou, Thèse pharm., Dakar 2008. Nº 70 